\title{
Ring chromosome 11 with KMT2A (MLL; 11q23) amplification and deletion in a patient with acute myeloid leukemia
}

Stephanie Liu*, Tahmeena Ahmed, Yupo Ma, Rajarsi Gupta, Theresa Mercado, Paula Fernicola, Daniel Tully, Erin Knorr and Ann-Leslie Zaslav

*Correspondence: stephanie.liu@stonybrookmedicine.edu

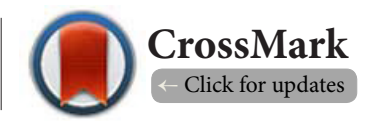

Stony Brook University Hospital, Department of Pathology, 101 Nicolls Rd, Level 2, Stony Brook, NY 11974, USA.

\begin{abstract}
Background: Chromosome abnormalities are significant in the diagnosis, prognosis, and treatment of patients with hematologic malignancies. Rearrangements of the mixed lineage leukemia gene (KMT2A; i.e. MLL) can occur in acute myeloid leukemia cases and are associated with a poor prognosis in all leukemias. Methods: We report a case of a 78 year old female with a past medical history of congestive heart failure, who presented with severe anemia (hemoglobin level of $6.4 \mathrm{~g} / \mathrm{dL}$ ), thrombocytopenia (platelet count of $57 \mathrm{k} / \mu \mathrm{L}$ ), and $17 \%$ blasts in the peripheral blood.

Results: The bone marrow core biopsy showed sheets of blasts consistent with acute myeloid leukemia with minimal maturation (AML M1). Flow cytometry showed a myeloid phenotype and expressed CD 33, 34, 38, 15, 135, 123, and 117. Cytogenetics showed a complex karyotype and G-banded chromosome analysis performed on bone marrow revealed: 47 52,X,-X,del(5)(q13q33), +6, +8, del(11) $(q 21 q 23.1),+13, \operatorname{der}(16) \mathrm{t}(16 ; 18)(\mathrm{p} 10 ; \mathrm{q} 10), \mathrm{i}(17)(\mathrm{q} 10),+1 \sim 3 \mathrm{r}(11)(\mathrm{p} 15 \mathrm{q} 23)[\mathrm{cp} 15]$. These cells had 1 to 3 ring chromosomes with amplified KMT2A and a deletion of KMT2A on one of the chromosome 11 homologs. FISH identified a deletion of the 5q31 (EGR1) region of chromosome 5, three copies of 8q21 (RUNX1T1), and 1-3 copies of 11q23 (KMT2A).

Conclusions: KMT2A amplification with ring chromosome 11 has been rarely reported. However, ring chromosome 11 in conjunction with the deletion of $11 \mathrm{q}$ (KMT2A) to our knowledge has never been reported. The patient completed Cycle 1 of Decitabine (5-aza-2'-deoxycytidine) but had to discontinue chemotherapy due to multiple complications, and subsequently expired in home hospice.
\end{abstract}

Keywords: KMT2A (11q23), MLL, ring chromosome 11, complex karyotype

\section{Introduction}

Chromosome abnormalities are significant in the diagnosis, prognosis, and treatment of patients with hematologic malignancies. Acute myeloid leukemia (AML) comprises about $80 \%$ of acute leukemias in adults, with an incidence of 3 to 5 cases per 100,000 people in the United States. About half of all AML patients will have at least one chromosomal abnormality [1]. Testing for these abnormalities is performed by standard and molecular cytogenetic techniques; for example, fluorescence in situ hybridization (FISH).

The most common chromosomal abnormalities in AML are translocations, followed by deletions, duplications, and rarely, ring chromosomes [2]. Ring chromosomes are structural abnormalities, formed when the ends of the chromosomes are lost and the short and long arms of the chromosome fuse.
Ring chromosomes involving 11q23 (KMT2A; i.e., MLL) have rarely been reported in AML. Rearrangements of $11 \mathrm{q} 23$ are associated with a poor prognosis in all leukemias [2]. Here we report a 78 year old female with AML that who had an unusual rearrangement of KMT2A, as well as additional complex chromosomal rearrangements.

\section{Case presentation}

We report a case of a 78 year old female with a medical history of hypertension and congestive heart failure. She was status post pacemaker and defibrillator implantation from an unknown date. The patient presented to an outside hospital with complaints of sudden retrosternal chest pain radiating to the back, shortness of breath, and exertional dyspnea. She also had progressive fatigue for two weeks. Due to her past 
medical history and presenting symptoms, the preliminary differential diagnoses at the outside hospital included acute coronary syndrome vs. demand ischemia vs. aortic dissection. Her first set of troponins was noted to be negative.

A complete blood count and differential diagnosis showed anemia and circulating blasts. After evaluation by the Hematology/Oncology team at the outside hospital, it was decided to transfer her to Stony Brook University Hospital (SBUH) in September 2015 for further management. Upon admission, she had severe anemia (hemoglobin level of $6.4 \mathrm{~g} / \mathrm{dL}$ [12-16], hematocrit of 19\% [37-47]), thrombocytopenia (platelet count of $57 \mathrm{k} / \mu \mathrm{L}$ [150-350]), and $17 \%$ blasts in the peripheral blood [0]. A manual review of her blood smear confirmed the presence of blasts, occasional immature monocytes, and small, hypolobated, hypogranular neutrophils (Figure 1). The patient was evaluated by our Hematology/Oncology team, and a bone marrow biopsy was performed.

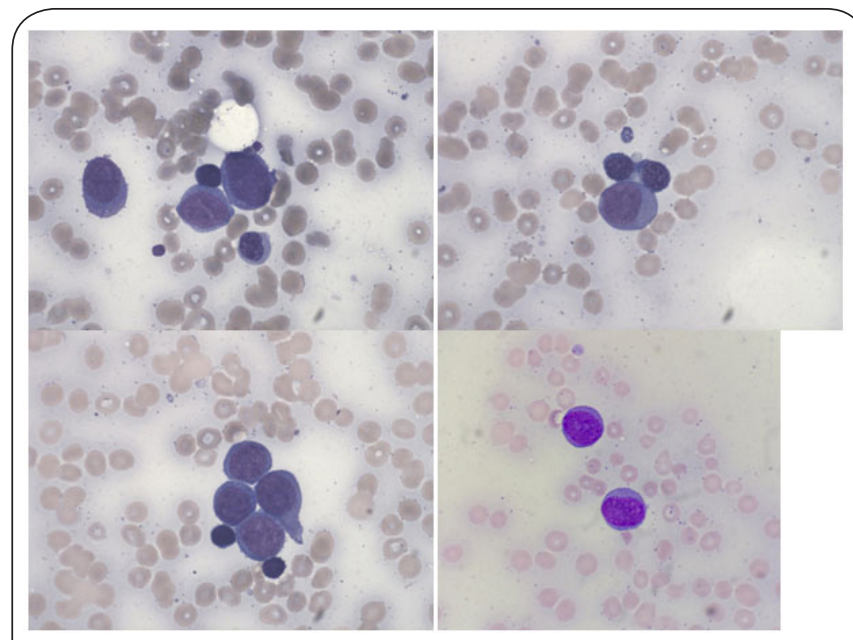

Figure 1. Blood smear: manual review of blood smear confirmed the presence of blasts, occasional immature monocytes, and small, hypolobated, hypogranular neutrophils.

\section{Materials and methods}

Conventional cytogenetic using $\mathrm{G}$ banding analysis of metaphase chromosomes and fluorescence in situ hybridization (FISH) were performed using standard techniques on bone marrow. G-banding analysis was performed on 15 metaphases from a 24 hour culture (Figure 2).

FISH analysis was performed on 200 nuclei and on an additional 10 metaphase cells (Figures 3 and 4). FISH probes used include LSI 5q EGR1/D5S23, D5S721 (5q31, 5p15.2), LSI RUNX1/RUNX1T1 (8q21.3, 21q22), LSI MLL DC BAR (11q23), and LSI D20S108 (20q12). All probes were supplied by Abbott Molecular, Des Plaines, IL USA.

Flow cytometry (Figure 5) was performed using a Becton Dickinson FACSCalibur using BD anti-CD33 (340679) and anti-CD34 (340667).

Blood counts were performed using a Sysmex XN-10. A
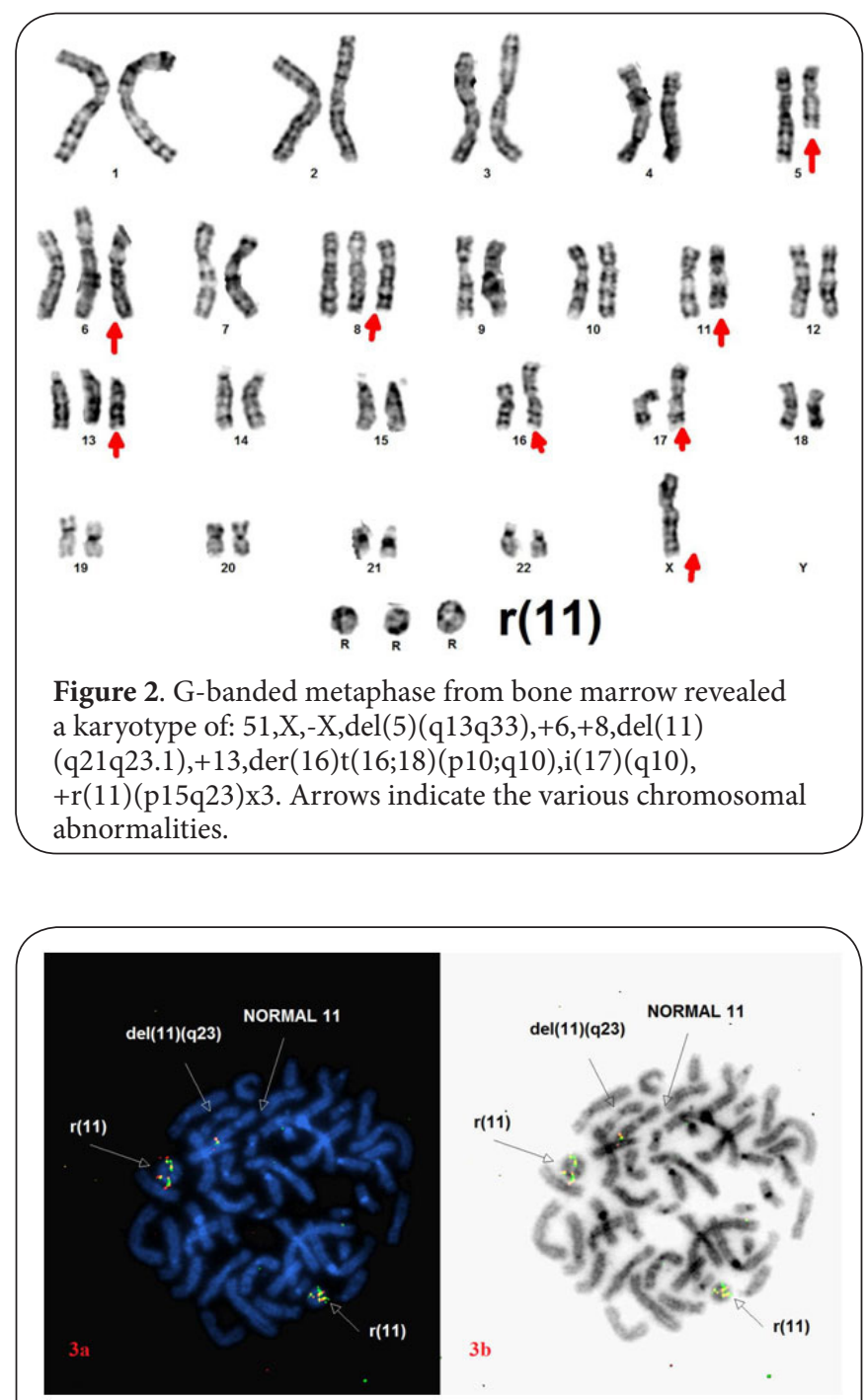

Figure 3. Metaphase FISH analysis was performed using the LSI MLL DC BAR (11q23) probe and demonstrated amplified copies of 11q23 (KMT2A) on the ring chromosomes and a deletion of 11q23 (KMT2A) on the del (11)(q23). (a): DAPI (4-6-diamidino-2-phenylindole), (b): Reverse DAPI image of the same metaphase.

blood smear was manually evaluated by a hematologist (Figure 1).

Molecular testing was performed by extracting the RNA from peripheral blood and reverse transcribed into CDNA. Amplification by real time PCR was performed with the BCR$A B L$ quantification kit from Roche Diagnostics. The control used was an amplification of the housekeeping gene, G6PDH. Results are reported as a ratio of $B C R-A B L$ to G6PDH transcript.

\section{Results}

The bone marrow core biopsy showed $>90 \%$ cellularity with sheets of blasts occupying the marrow space (Figure 6), confirming a diagnosis of acute myeloid leukemia with 


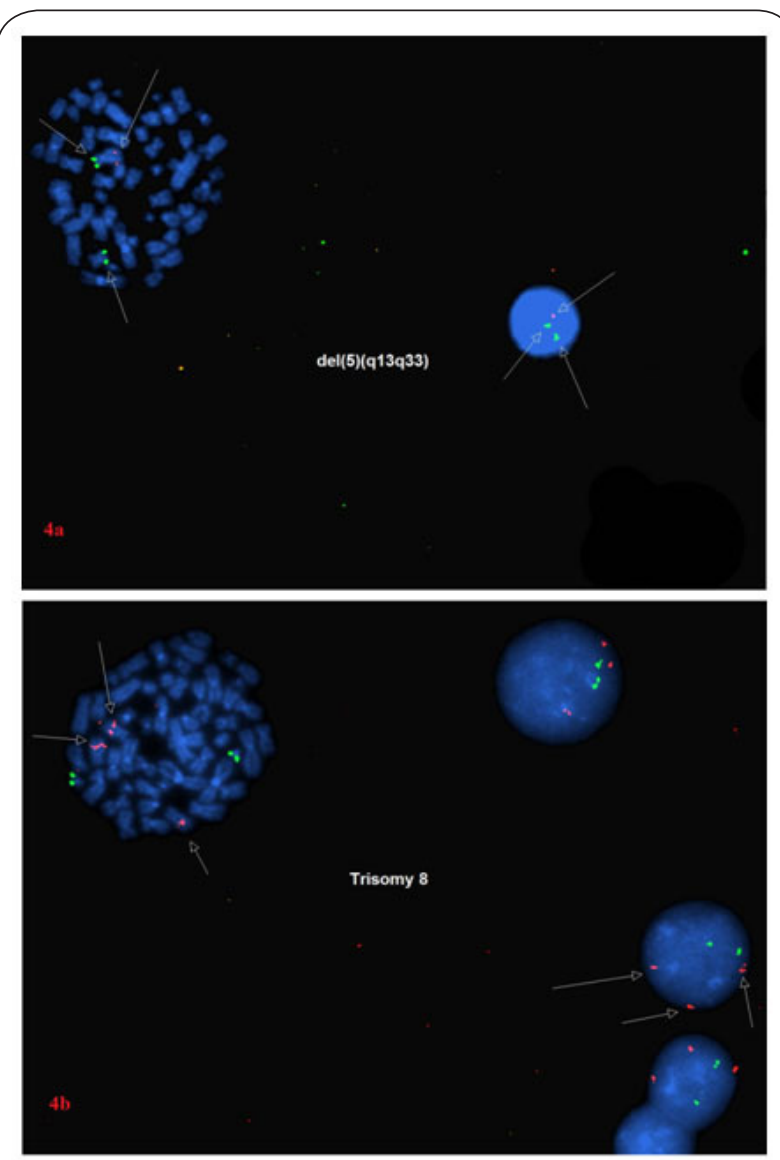

Figure 4. (a) Nuclear and metaphase FISH was performed using the LSI 5q EGR1/D5S23, D5S721 probes (5q31, orange; $5 \mathrm{p} 15.2$, green). Arrows indicate both chromosome 5 homologs. The del (5)(q31) is missing the orange signal in both the metaphase and the nucleus.

(b): Nuclear and metaphase FISH was performed using the LSI RUNX1/RUNX1T1 probes (8q21.3, orange; 21q22, green). Arrows indicate three copies of $8 \mathrm{q} 21.3$ in both the metaphase and the nuclei.

minimal maturation (AML M1, according to FAB classification [3]). Dyserythropoiesis was present, with megaloblastoid change and binuclear erythrocytes. Lymphoid aggregates and extrinsic cells were not present. Marrow fibrosis was not seen. Megakaryocytes were present, but reduced in number. Stainable iron was decreased. Spicules were not present on the aspirate or touch preparation.

Flow cytometry showed a myeloid immunophenotype, with the presence of an intermediate to large sized myeloid blast cell population (Figure 5). The leukemic blasts expressed CD33, CD34, CD38, CD15, CD135, CD123, CD11b, and some CD117. There was no significant expression of $B$ cell or $T$ cell markers. The results, according to FAB classification [3], were consistent with AML M1. Molecular genetic testing on the patient's peripheral blood did not detect the BCR-ABL1 fusion transcript.

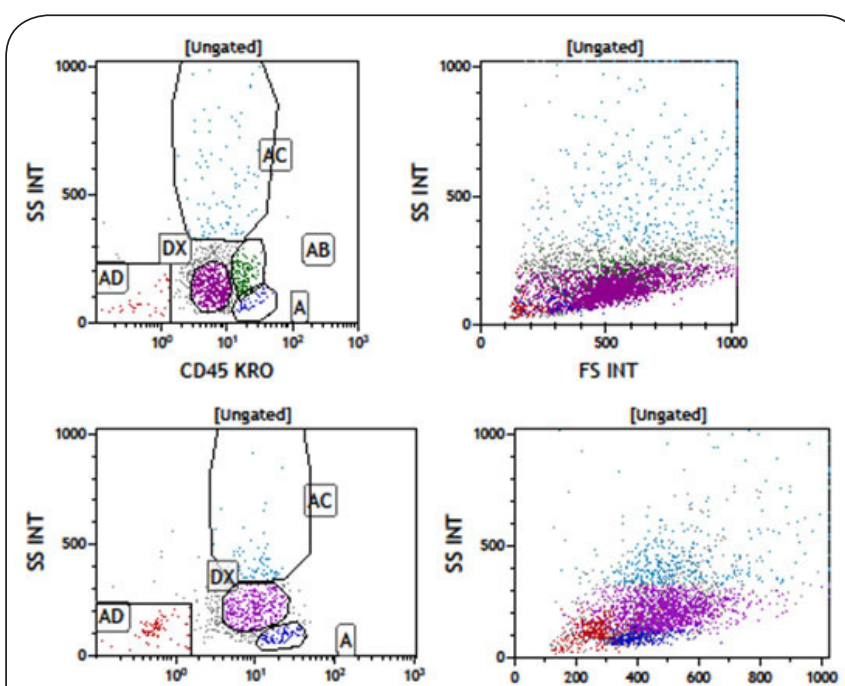

Figure 5. Bone marrow flow cytometry: Flow cytometry showed a myeloid immunophenotype, with the presence of an intermediate to large sized myeloid blast cell population. The leukemic blasts expressed CD33, CD34, CD38, CD15, CD135, CD123, CD11b, and some CD117. There was no significant expression of $\mathrm{B}$ cell or $\mathrm{T}$ cell markers. The results, according to FAB classification, were consistent with AML M1.

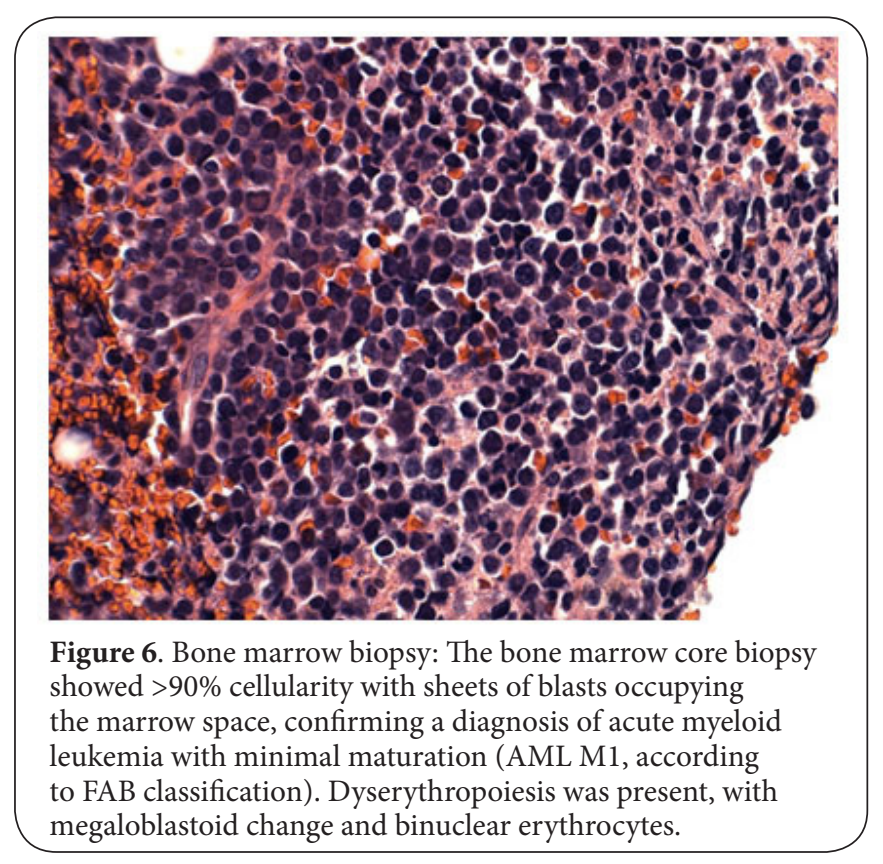

Cytogenetics showed a complex karyotype (Figure 2). G-banded chromosome analysis of bone marrow revealed $47 \sim 52, \mathrm{X}$,$X, \operatorname{del}(5)(q 13 q 33),+6,+8, \operatorname{del}(11)(q 21 q 23.1),+13, \operatorname{der}(16) t(16 ; 18)$ (p10;q10),i(17)(q10),+1 3r(11)(p15q23)[cp15]. All cells had an abnormal number of chromosomes, between 47 to 52 chromosomes per cell. Some cells were missing one $X$ chromosome $(-X)$. Trisomy 6,8 , and 13 were present in all cells. Most cells had a deletion of part of the long arm of one chromosome 
5 homolog, del(5)(q13q33). The deletion of part of the long arm of one chromosome 11 homolog, del(11)(q21q23.1), was also seen in most cells. Another abnormality was a derivative chromosome 16, resulting from an unbalanced whole arm translocation between the short arm of one chromosome 16 homolog and the long arm of chromosome $18, \operatorname{der}(16) t(16 ; 18)$ (p10;q10). An isochromosome of the long arm of chromosome 17, i(17)(q10), was also present in most cells. One to three ring chromosomes were seen on all cells. These ring chromosomes contained an amplification of KMT2A, and one of the chromosome 11 homologs had a deletion of KMT2A. All other chromosomes appeared to be structurally normal.

Interphase FISH on 200 nuclei confirmed a deletion of the 5 q31 (EGR1) region of chromosome 5 in $92 \%$ of the nuclei (Figure 4a). Three copies of 8q21 (RUNX1T1) were present on $81 \%$ of nuclei (Figure $4 \mathbf{b}$ ). There were 1 to 3 copies of $11 \mathrm{q} 23$ in $88.5 \%$ of nuclei (KMT2A, Figure 3 ). FISH results for chromosomes 7, 20, and 21 were normal.

Upon arrival to SBUH, the patient developed a neutropenic fever and her blood cultures were found to be positive for Staphylococcus hemolyticus. Vancomycin resistant Enterococci was found in her urine culture, and methicillin-resistant Staphylococcus aureus was found in her sputum culture. The patient was started on cycle 1 of Decitabine, but suffered acute respiratory failure after the third dose. She was intubated and the chemotherapy cycle was completed. Ten days after this, she continued deteriorating, and was given a tracheostomy and percutaneous endoscopic gastrostomy (PEG) tube.

One week later, she suffered a small bowel obstruction, and her CT scan showed a possible aspiration pneumonia. Pseudomonas was identified as the cause. An additional chest $x$-ray was performed which showed worsening diffuse pneumonia bilaterally. A subsequent flow cytometry analysis of peripheral blood did not detect evidence of AML. She was re-evaluated by the Hematology/Oncology service and was no longer considered to be a candidate for chemotherapy. The patient's family decided to pursue palliative care, and she was discharged to home hospice approximately two months after her initial presentation, where she expired.

\section{Discussion}

Acute myeloid leukemia is the most common acute leukemia in middle aged and older adults. The median age is 60 , with an incidence of 10:100,000 in people over 60 years [2]. The male to female incidence is $5: 3$, and it is slightly more common in non-Hispanic whites than in other races [1]. Although AML can be associated with certain environmental factors, genetic abnormalities, and other benign or malignant processes, most cases are idiopathic. Patients commonly present with symptoms similar to those seen in an acute infection. These symptoms are related to pancytopenia and include infections, fatigue, and hematologic manifestations such as hemorrhage of mucosal membranes, pallor, menorrhagia, ecchymoses, and epistaxis $[\mathbf{1 , 2}]$. Bone pain is not common, but has been reported in the sternum and long bones [1].

Approximately $50 \%$ of all AML patients will have cytogenetic abnormalities [1]. Abnormalities include balanced translocations, where there is an even exchange of material. Translocations can also be unbalanced, where the exchange of chromosomal material is unequal, resulting in extra or missing genetic material. Deletions are the loss of the part of a chromosome, which can lead to partial monosomy. Amplifications, or duplications, are additional copies of a chromosome, and can cause partial trisomy. Inversions are seen in chromosomes with two breaks that result in rotation of the genetic material. A karyotype containing three or more abnormalities is referred to as complex, and occurs in up to $40 \%$ of AML in adults [4].

Our patient had a number of abnormalities commonly seen in AML: trisomy 8 , deletion of $5 q$, trisomy 13 , (der) $16 p$, isochromosome 17, and rearrangement of 11q23. Trisomy 8 is the most common abnormality in AML, occurring in $10 \%$ to $15 \%$ of AML cases [4]. Schoch et al. reported significantly upregulated levels of the PTK2B, CNOT7, and LYPLA1 genes in AML patients with trisomy 8 . This shows that the gain of an extra chromosome causes overexpression of certain genes [5]. However, having trisomy 8 as a sole karyotypic abnormality is thought not to be sufficient to initiate leukemia. Other genetic changes must take place, since trisomy 8 is commonly associated with multiple chromosomal abnormalities $[4,5]$.

Deletion of $5 q$ occurs in $5 \%$ of $A M L$, and the incidence increases with increased age. This can either be a sole abnormality or in association with a complex karyotype [4]. The deletion of $5 q$ is associated with resistance to chemotherapy, so patients with a $5 q$ deletion are considered high risk [6].

Trisomy 13 is seen in $2 \%$ to $3 \%$ of AML karyotypes, and was the sole abnormality in $0.7 \%$ of cases [7]. RUNX1 mutations and increased FLT3 expression were shown to exist in AML cases with trisomy 13 [8].

Rearrangements of chromosome 16 are seen in $0.3 \%$ to $3.2 \%$ of AML translocations and inversions, respectively [9]. This involves the rearrangement of the core binding factor beta (CBF $\beta$ ) gene, which also controls the RUNX1 protein [10].

Isochromosome 17, $\mathrm{i}(17)(\mathrm{q} 10)$, is actually an isodicentric abnormality; the chromosome has two $17 q$ centromeres fused together, effectively producing a deletion of $17 p$ [4]. This results in the loss of the tumor suppressor gene TP53 [11]. It is commonly seen in association with other genetic abnormalities, such as the del $(5 q),+8$, and +13 in our patient. Isochromosome 17 is not specific to AML either; it can occur in other hematologic malignancies, myeloproliferative neoplasms, and solid tumors [12].

Rearrangements of the mixed lineage leukemia gene (KMT2A; i.e., MLL) have been reported in approximately $7.8 \%$ of AML cases and are associated with a poor prognosis in all leukemias [13]. Translocations involving the 11q23 chromosomal region can disrupt the KMT2A gene [14]. The KMT2A protein is a histone methyltransferase which assembles in protein complexes that regulate gene transcription via 
chromatin remodeling [15]. These proteins include the Hox genes (Homeobox domain gene), which play a critical role in development as well as hematopoiesis. Both the expression of Hox genes and the up-regulation of KMT2A fusion proteins are needed to cause leukemia [15]. However, the KMT2A gene is not specific to AML. KMT2A translocations can be involved in acute lymphoblastic, minimally differentiated, or mixedlineage leukemias, along with myelodysplastic syndrome [14].

Most KMT2A abnormalities involve translocations and fusions with various partners, with 45 partners in AML. However, Andersen et al. reported extrachromosomal amplifications of KMT2A [16]. The patients were elderly and had a whole or partial deletion of chromosome $5 q$. Patients with a deletion of $7 q$ and unaffected chromosomes 5 did not have KMT2A amplification. Some patients gained duplications or amplifications on a derivative chromosome 11. Others gained a whole chromosome 11 without additional genetic material from other chromosomes. Eleven out of 12 of these patients with KMT2A duplication or amplification were previously treated with an alkylating agent, suggesting a possible relation [16]. The gain of $11 \mathrm{q}$ is seen in fewer than $2 \%$ of AML, and the cases have a poor prognosis [17].

Deletion of the KMT2A gene is rare in AML. Ma et al. reported 9 cases of del(11q) in a total of $819 \mathrm{AML}$ cases, at 1.1\% [18]. Some cases had a whole chromosome deletion, while others showed the deleted chromosome 11 material translocated to other chromosomes. The mechanism causing the deletion has not been identified [18].

Ring chromosomes are also rare, and result from one of two mechanisms. Breaks can occur on each arm of one chromosome, with subsequent reattachment at the break sites [19]. Breaks on the same arm can also take place, but this is much rarer. The other mechanism involves dysfunctional telomeres, which can also cause the ends to fuse without loss of genetic material. Shortening of telomeres may result in detachment of the ends, causing the ends to become prone to recombination [19].

Ring chromosomes are very unstable. During mitosis, the rings may become entangled, broken, doubled, and/or disrupted. Daughter cells that arise would then be aneuploid. This causes chromosome instability and a "dynamic mosaicism" [20]. This was seen in our patient, where the number of rings ranged from 1 to 3 per cell and also showed varying appearances. The $\mathrm{r}(11)$ also demonstrated amplification of KMT2A (Figures 2 and $\mathbf{3}$ ).

Ring chromosomes are commonly seen in sarcomas; for example, they are present in $70 \%$ of dermatofibrosarcoma protuberans [19], and all reported cases of parosteal osteosarcoma [21]. They are also seen in various types of bone and soft tissue tumors, including lipomas, leiomyomas of the uterus, and fibrosarcomas $[\mathbf{4 , 2 2}]$. The presence of a ring chromosome in the setting of AML constitutes a complex karyotype and a poor prognosis. The first report of a ring chromosome in AML was in 1962, although the chromosome was not speci- fied [23]. KMT2A amplification with ring chromosome 11 has been rarely reported in $A M L[16,17,24,25]$. KMT2A deletion in association with ring chromosome 11, to our knowledge, has never been reported.

Analysis of G-banded metaphases demonstrated that the ring chromosome(s) 11 seen in our patient did not appear to have additional material from other chromosomes. The partial deletion of 11q did not involve a translocation, which was confirmed by FISH. All cells had at least one ring chromosome 11 with amplification of KMT2A and only one normal chromosome 11. The derivative chromosome $11 \mathrm{did}$ not contain KMT2A, confirming the cytogenetic results (Figure 2).

Other chromosome abnormalities not typically associated with AML include the loss of the $X$ chromosome and trisomy 6. Deletion of the $X$ chromosome in association with other abnormalities is seen in $1.5 \%$ of AML and myelodysplastic syndromes (MDS) patients [25]. Trisomy 6 occurs more commonly in skin tumors, such as basal cell carcinomas, melanocytic nevi, and merkel cell carcinomas, than in AML [26]. In AML, trisomy 6 has been reported in association with AML M1 [24], as seen in our patient.

The complexity of this patient's karyotype, specifically with the multiple ring chromosomes, amplified KMT2A, and the deletion of 11q23, have rarely been reported [27-31]. These abnormalities, as well as the additional complex rearrangement, were significant in the evolution of this patient's disease and conferred a very poor prognosis.

\section{Conclusion}

Our patient presented with a complex karyotype, with abnormalities of chromosomes X, 5, 6, 8, 13, 16, 17, and $11 q 23$. Ring chromosome 11 and amplification of KMT2A (11q23) in conjunction with the deletion of KMT2A to our knowledge has never been reported. The complex karyotype was significant in the evolution of her disease and resulted in an extremely poor prognosis. She completed Cycle 1 of decitabine but had multiple complications, including pneumonia requiring intubation and sepsis, and was discharged to home hospice. This case study demonstrated the difficulty of effectively treating patients with complex chromosome abnormalities. The identification of additional AML cases with complex karyotypes that have amplification of KMT2A will help determine their impact in the diagnosis and prognosis for these types of patients.

\section{Competing interests}

The authors declare that they have no competing interests.

\section{Acknowledgements}

We would like to thank the following people for their assistance: Marc Golightly, PhD: Flow cytometry, Silvia Spitzer, PhD: Molecular genetics, Lisa Senzel MD, PhD: Hematology. 
Liu et al. Hematology and Leukemia 2017,

http://www.hoajonline.com/journals/pdf/2052-434X-5-3.pdf

doi: $10.7243 / 2052-434 X-5-3$

Authors' contributions

\begin{tabular}{|l|c|c|c|c|c|c|c|c|c|}
\hline Authors' contributions & SL & TA & YM & RG & TM & PF & DT & EK & AZ \\
\hline Research concept and design & $\checkmark$ & $\checkmark$ & -- & -- & -- & -- & -- & -- & $\checkmark$ \\
\hline Collection and/or assembly of data & $\checkmark$ & -- & -- & $\checkmark$ & $\checkmark$ & $\checkmark$ & $\checkmark$ & $\checkmark$ & $\checkmark$ \\
\hline Data analysis and interpretation & $\checkmark$ & -- & -- & -- & -- & -- & -- & -- & $\checkmark$ \\
\hline Writing the article & $\checkmark$ & -- & -- & -- & -- & -- & -- & -- & $\checkmark$ \\
\hline Critical revision of the article & $\checkmark$ & $\checkmark$ & $\checkmark$ & -- & -- & -- & -- & -- & $\checkmark$ \\
\hline Final approval of article & & $\checkmark$ & $\checkmark$ & -- & -- & -- & -- & -- & $\checkmark$ \\
\hline Statistical analysis & $\checkmark$ & -- & -- & $\checkmark$ & $\checkmark$ & $\checkmark$ & $\checkmark$ & $\checkmark$ & $\checkmark$ \\
\hline
\end{tabular}

Publication history

Editor: Sabrina Tosi, Brunel University, UK.

Received: 30-Jun-2017 Final Revised: 25-Jul-2017

Accepted: 30-Aug-2017 Published: 08-Sep-2017

\section{References}

1. Schiffer CA and Anastasi J. Clinical manifestations, pathologic features, and diagnosis of acute myeloid leukemia. UpToDate. In A. G. Rosmarin (Ed.). 2017.

2. McPherson RA, Pincus MR and Henry JB. Henry's clinical diagnosis and management by laboratory methods. Philadelphia, PA: Elsevier Saunders. 2011.

3. Swerdlow SH, Campo E and Harris NLet al. WHO Classification of Tumours of Haematopoietic and Lymphoid Tissues. Lyon, France: IARC Press; 2008.

4. Heim S and Mitelman F. (Eds). Cancer Cytogenetics: Chromosomal and Molecular Genetic Aberrations of Tumor Cells. ( $4^{\text {th }}$ Ed.). Chichester, UK: John Wiley \& Sons, Ltd. 2015.

5. Schoch C, Kohlmann A, Dugas M, Kern W, Hiddemann W, Schnittger S and Haferlach T. Genomic gains and losses influence expression levels of genes located within the affected regions: a study on acute myeloid leukemias with trisomy $\mathbf{8 , 1 1}$, or 13 , monosomy 7 , or deletion $5 q$. Leukemia. 2005; 19:1224-8. | Article | PubMed

6. Samuels BL, Larson RA, Le Beau MM, Daly KM, Bitter MA, Vardiman JW, Barker CM, Rowley JD and Golomb HM. Specific chromosomal abnormalities in acute nonlymphocytic leukemia correlate with drug susceptibility in vivo. Leukemia. 1988; 2:79-83. | PubMed

7. Mesa RA, Hanson CA, Ketterling RP, Schwager S, Knudson RA and Tefferi A. Trisomy 13: prevalence and clinicopathologic correlates of another potentially lenalidomide-sensitive cytogenetic abnormality. Blood. 2009; 113:1200-1. | Article | PubMed

8. Dicker F, Haferlach C, Kern W, Haferlach T and Schnittger S. Trisomy $\mathbf{1 3}$ is strongly associated with AML1/RUNX1 mutations and increased FLT3 expression in acute myeloid leukemia. Blood. 2007; 110:1308-16. | Article | PubMed

9. Eghtedar A, Borthakur G, Ravandi F, Jabbour E, Cortes J, Pierce S, Kantarjian $\mathrm{H}$ and Garcia-Manero $\mathrm{G}$. Characteristics of translocation (16;16)(p13;q22) acute myeloid leukemia. Am J Hematol. 2012; 87:3178. | Article | PubMed Abstract | PubMed FullText

10. Aventin A, Espadaler M, Casas S, Duarte J, Nomdedeu J and Sierra J. Chromosome 16 inversion-associated translocations in acute myeloid leukemia elucidated using a dual-color CBFB DNA probe. Cancer Genet Cytogenet. 2002; 134:142-4. | Article | PubMed

11. Kanagal-Shamanna R, Bueso-Ramos CE, Barkoh B, Lu G, Wang S, Garcia-Manero G, Vadhan-Raj S, Hoehn D, Medeiros LJ and Yin CC. Myeloid neoplasms with isolated isochromosome 17q represent a clinicopathologic entity associated with myelodysplastic/ myeloproliferative features, a high risk of leukemic transformation, and wild-type TP53. Cancer. 2012; 118:2879-88. | Article | PubMed

12. Mertens F, Johansson B and Mitelman F. Isochromosomes in neoplasia. Genes Chromosomes Cancer. 1994; 10:221-30. I PubMed

13. Ibrahim S, Estey EH, Pierce S, Glassman A, Keating M, O'Brien S, Kantarjian HM and Albitar M. 11q23 abnormalities in patients with acute myelogenous leukemia and myelodysplastic syndrome as detected by molecular and cytogenetic analyses. Am J Clin Pathol. 2000; 114:793-7. | Article | PubMed

14. Mitterbauer-Hohendanner $\mathrm{G}$ and Mannhalter $\mathrm{C}$. The biological and clinical significance of MLL abnormalities in haematological malignancies. Eur J Clin Invest. 2004; 34 Suppl 2:12-24. | Article | PubMed

15. Hess JL. MLL: a histone methyltransferase disrupted in leukemia. Trends Mol Med. 2004; 10:500-7. | Article | PubMed

16. Andersen MK, Christiansen DH, Kirchhoff M and Pedersen-Bjergaard J. Duplication or amplification of chromosome band 11q23, including the unrearranged MLL gene, is a recurrent abnormality in therapy-related MDS and AML, and is closely related to mutation of the TP53 gene and to previous therapy with alkylating agents. Genes Chromosomes Cancer. 2001; 31:33-41. | Article | PubMed

17. Andersen MK, Christiansen DH and Pedersen-Bjergaard J. Amplification or duplication of chromosome band 21q22 with multiple copies of the AML1 gene and mutation of the TP53 gene in therapy-related MDS and AML. Leukemia. 2005; 19:197-200. | Article | PubMed

18. Ma SK, Wan TS, Au WY, Fung LF, So CK and Chan LC. Chromosome 11q deletion in myeloid malignancies. Leukemia. 2002; 16:953-5. | Article | PubMed

19. Gisselsson D. Ring chromosomes: vicious circles at the end and beginning of life. Atlas Genet CytogenetOncolHaematol. 2001.

20. Gardner RM, Sutherland GR and Shaffer LG. Chromosome Abnormalities and Genetic Counseling. (4th Ed.). New York: Oxford University Press. 2011.

21. Szymanska J, Mandahl N, Mertens F, Tarkkanen M, Karaharju E and Knuutila S. Ring chromosomes in parosteal osteosarcoma contain sequences from 12q13-15: a combined cytogenetic and comparative genomic hybridization study. Genes Chromosomes Cancer. 1996; 16:314. | Article | PubMed

22. Donner LR. Cytogenetics of tumors of soft tissue and bone. Implication for pathology. Cancer Genet Cytogenet. 1994; 78:115-26. | PubMed

23. Sandberg AA, Ishihara T, Crosswhite LH and Hauschka TS. Chromosomal dichotomy in blood and marrow of acute leukemia. Cancer Res. 1962; 22:748-56. | Article | PubMed

24. Bacher U, Schanz J, Braulke F and Haase D. Rare cytogenetic abnormalities in myelodysplastic syndromes. Mediterr J Hematol Infect Dis. 2015; 7:e2015034. | Article | PubMed Abstract | PubMed FullText

25. Zatkova A, Ullmann R, Rouillard JM, Lamb BJ, Kuick R, Hanash SM, Schnittger S, Schoch C, Fonatsch C and Wimmer K. Distinct sequences on 11q13.5 and 11q23-24 are frequently coamplified with MLL in complexly organized 11q amplicons in AML/MDS patients. Genes Chromosomes Cancer. 2004; 39:263-76. | Article | PubMed

26. Larsimont $D$ and Verhest $A$. Chromosome 6 trisomy as sole anomaly in a primary Merkel cell carcinoma. Virchows Arch. 1996; 428:305-9. | PubMed

27. Park JP, Ladd SL, Ely P, Weiner NJ, Wojiski SA, Hawk AB, Noll WW and Mohandas TK. Amplification of the MLL region in acute myeloid leukemia. Cancer Genet Cytogenet. 2000; 121:198-205. | Article | PubMed

28. Streubel B, Valent $P$, Jager $U$, Edelhauser $M$, Wandt $H$, Wagner $T$, Buchner $\mathrm{T}$, Lechner $\mathrm{K}$ and Fonatsch C. Amplification of the MLL gene on double minutes, a homogeneously staining region, and ring chromosomes 
Liu et al. Hematology and Leukemia 2017,

http://www.hoajonline.com/journals/pdf/2052-434X-5-3.pdf

in five patients with acute myeloid leukemia or myelodysplastic syndrome. Genes Chromosomes Cancer. 2000; 27:380-6. | PubMed

29. Tyybakinoja A, Saarinen-Pihkala U, Elonen E and Knuutila S. Amplified, lost, and fused genes in 11q23-25 amplicon in acute myeloid leukemia, an array-CGH study. Genes Chromosomes Cancer. 2006; 45:257-64. | Article | PubMed

30. Wang SA, Abruzzo LV, Hasserjian RP, Zhang L, Hu Y, Zhang Y, Zhao M, Galili N, Raza A, Medeiros LJ, Garcia-Manero G and Miranda RN. Myelodysplastic syndromes with deletions of chromosome 11q lack cryptic MLL rearrangement and exhibit characteristic clinicopathologic features. Leuk Res. 2011; 35:351-7. | Article | PubMed

31. Poppe B, Vandesompele J, Schoch C, Lindvall C, Mrozek K, Bloomfield CD, Beverloo HB, Michaux L, Dastugue N, Herens C, Yigit N, De Paepe A, Hagemeijer $A$ and Speleman F. Expression analyses identify MLL as a prominent target of 11q23 amplification and support an etiologic role for MLL gain of function in myeloid malignancies. Blood. 2004; 103:22935. | Article | PubMed

\section{Citation:}

Liu S, Ahmed T, Ma Y, Gupta R, Mercado T, Fernicola P, Tully D, Knorr E and Zaslav A-L. Ring chromosome 11 with KMT2A (MLL; 11q23) amplification and deletion in a patient with acute myeloid leukemia. Hematol Leuk. 2017; 5:3. http://dx.doi.org/10.7243/2052-434X-5-3 